・保护论坛・

\title{
关于提高物种划分合理性的意见
}

\author{
洪德 ${ }^{*}$ \\ (中国科学院植物研究所系统与进化植物学国家重点实验室, 北京 100093)
}

\section{Opinion of raising rationality in species delimitation}

\begin{abstract}
Deyuan Hong $^{*}$
State Key Laboratory of Systematic and Evolutionary Botany, Institute of Botany, Chinese Academy of Sciences, Beijing 100093

这是我在The Flora of Pan-Himalaya (泛喜马拉雅植物志)第二次全体国内作者会议上关于物种划分问 题的发言的结尾部分。我认为物种划分是分类学(狭义)的核心内容, 所以我以辐冠参属(Pseudocodon)的鸡 蛋参复合群(Pseudocodon convolvulaceus complex)和䓎药属(Paeonia)的滇牡丹复合群(Paeonia delavayi complex)为例，重点陈述了我处理物种划分的思路和做法。发言后，不少听众很关注我的十点看法，更有 些同事希望我发表出来。我感谢同事们的好意。物种是生物多样性的基本单元, 所以我把发言稍加修改后 在《生物多样性》发表, 表达我对物种划分的观点。我是把这十点意见作为讨论和点评的靶子发表的, 希 望能引起同行的关注、讨论和点评。
\end{abstract}

\section{1 分类学家要有宽而厚的生物学基础}

分类学(taxonomy)有广义和狭义两种概念：广 义的分类学研究的内容包括分类(classification)、系 统发生(phylogeny)和进化(evolution), 狭义的只包 括分类研究。但是, 不管广义的还是狭义的, 分类 学都是一门高度综合的学科。从事分类研究的学者 首先必须是一位生物学家, 特别要有遗传学、发育 生物学的知识背景, 要深刻理解生物的变异和进 化。物种划分是分类研究的核心内容, 因此还必须 具有深厚的居群生物学(population biology)知识。

\section{2 性状分析是分类的基本功}

物种划分依据性状，而性状有无分类价值、分 类价值高低, 要通过性状分析加以评价。因此性状 分析是分类研究的基本功。除了对前人应用的性状 要重新分析和评价外, 还要通过认真观察, 发现新 的性状。要分析性状的变异规律: 变异的诱因(遗传 变异还是环境饰变)、变异的幅度、变异的连续性和 间断性、趋同变异还是共衍征。特别要分析性状在 居群内、居群间和地区间的变异, 性状与海拔和环
境之间的关系等。只有性状分析到位，才能正确判 断性状的分类价值，才有合理的物种划分。

\section{3 树立居群概念，摒弃模式概念}

居群内可能有无数的基因型, 无数的类型; 居 群间也可能因生态环境和地理的原因存在差异, 要 充分理解诸如可塑性 (plasticity)、饰变(modification)、基因型(genotype)、突变体(variant)、多态现 象(polymorphism)、类型(form)、梯度变异(cline)、 生态型(ecotype)、宗(race)等等的内涵。不要把多态 现象和杂交混为一谈。要重视野外观察, 特别要注 重观察居群内变异。居群概念也体现在标本馆标本 的研究中。是否乐于观察和鉴定尽可能多的标本, 认真分析变异, 是否依据全部标本和野外观察资料 来描述物种和编制检索表，是两种概念的反映。

\section{4 吸纳新思想、新方法、新数据}

物种划分一定要理解并应用分子、基因组等先 进的研究思路、方法和数据。这方面研究产生的结 果可以为我们提供无可争辩的证据。当然也要看数 据的支持强度, 理性看待各类数据。

\footnotetext{
* 通讯作者 Author for correspondence. E-mail: hongdy@ibcas.ac.cn
} 


\section{5 综合手段不可缺}

染色体、花粉以及其他学科提供的数据和资料 都可能是很有价值的, 甚至是不可或缺的。分类研 究必须采用综合手段。

\section{6 一定要引入统计学方法}

一位先人说过, 不引入数学的方法, 任何一门 科学都是不精确的。物种划分中应用的性状很多是 数量性状, 而统计学是分析数量性状变异的最佳手 段。例如标准差分析可显示一个数量性状的变异在 两个或多个类群间是连续的还是间断的; 聚类分析 和主成分分析可以分析多个性状，展示类群间的关 系, 为物种区分还是归并提供佐证。用于统计学分 析的最好材料是居群取样, 标本也可以用。

\section{7 认识物种之间的形态表现}

一个自然的物种 (即“好种”)都会和近缘物种在 两个或多个性状上表现出变异的间断(数量性状则 是在统计上的间断)。这样的间断是基因流长期隔断 造成形态分化的结果。基因流隔断则是生殖隔离、 地理隔离或生态隔离的结果。因此, 这样划分的物 种反映了物种的基本生物学特性。切忌依据单个性 状划分物种。当然, 如何处理像竹子、蒲公英等多 为营养繁殖或无融合繁殖的植物, 仍是分类学的一 大挑战。

\section{8 尽可能查看模式标本}

查看模式标本的必要性在于: (1)可以避免张冠 李戴; (2)模式标本, 特别是早期的模式标本有可能 是混杂物, 即同号标本包含两个物种, 甚至一张台 纸上装着两个物种的标本。认真检查可澄清文献中 存在的物种问题。

\section{9 为分类学“正名”而努力}

分类学有“不好”的名声。国际上历来有“分裂 派”(splitter) 和 “归并派”(lumper)之称; 连著名的 Principles of Angiosperm Taxonomy (被子植物分类学 原理) (Davis \& Heywood, 1963)一书也说“分类学一 半是科学, 一半是艺术”。两年前, 我再次见到 Heywood先生。我问他, 过了 50 年, 对这一问题是否 改变了主意, 他说: 恩, 分类学 $51 \%$ 是科学! 中国植
物分类学界的名声更有问题。国际上一些植物分类 学家多次在杂志上批评我们是“分裂派”, 意指乱造 新种(Hedge et al, 1979; Nooteboom, 1992; Ferguson, 2003)。“分类不是科学”、“公说公有理, 婆说婆有理” 的说法在国内颇盛行。原因有三: (1)客观因素。分 类学的核心内容是研究生物多样性的基本单元, 即 物种, 而多样性可以说是“复杂”的代名词。(2)历史 造成。中国植物分类研究不仅历史短暂, 而且十分 艰难。《中国植物志》就是在客观条件、人才培养 和学术准备都不足的情况下启动和进行的。(3)客观 和主观共同作用的结果。在我国, 20 世纪 50 年代以 前是战乱, 在五、六十年代, 真正的遗传学被批为 “反动的、唯心的”。我们这一代, 甚至年轻一代的 不少人没有遗传学的背景, 缺乏生物变异和进化的 思想, 因而无意识地受模式概念的束缚。分类学无 疑是一门综合而复杂的科学, 在错误观念指导下研 究的结果可想而知。当然, 在正确思想指导下完成 的分类处理也可能因客观条件限制尚有不科学的 成份。我们的目标是树立正确观念, 创造或改善客 观条件, 运用科学的研究方法, 努力使研究结果越 来越接近科学。

\section{0 合理的物种划分是生物多样性研究、保 护和可持续利用的基础}

物种是生物多样性的基本单位, 可见物种划分 在生物学中的份量。物种划分要建立在理解物种的 基础上, 要有扎实的学科基础, 综合的思维和研究 方法。我们把《泛喜马拉雅植物志》定位为最高水 平的植物志, 已出版的三卷册已展示了这一点。我 希望我们所有作者加厚自身的生物学, 特别是遗传 学、居群生物学等的积淀, 运用综合的思维和研究 方法。只有这样, 我们才能为物种划分提供令人信 服的证据, 大大加重植物志中科学的份量。

\section{参考文献}

Davis PH, Heywood VH (1963) Principles of Angiosperm Taxonomy. Oliver \& Boyd, Edinburgh \& London.

Ferguson DK (2003) Book review. Systematic Botany, 28, 808-809.

Hedge IC, Lauener LA, Tan HK (1979) Book review. Notes Royal Botanic Garden Edinburgh, 37, 467-468.

Nooteboom HP (1992) A point of view on the species concept. Taxon, 41, 318-320. 